\title{
Mechanical and thermal characterization of a novel nanocomposite thermal interface material for electronic packaging
}

\author{
Shuangxi Sun ${ }^{1,2}$, Si Chen ${ }^{1,2}$, Xin Luo ${ }^{1,2}$, Yifeng Fu, ${ }^{2,3}$, Lilei Ye ${ }^{3}$, Johan Liu ${ }^{1,2}$ \\ 1SMIT Center, School of Automation and Mechanical Engineering, Key Laboratory of Advanced \\ Display and System Applications, Shanghai University, No 20, Chengzhong Road, 2nd Floor, \\ Chemical Building, Shanghai, zip code: 201800, China
}

2Electronics Material and Systems Laboratory, Department of Microtechnology and Nanoscience, Chalmers University of Technology, Kemivägen 9, Se 41296 Gothenburg, Sweden

3SHT - Smart High Tech AB, Aschebergsgatan 46, 41133 Gothenburg, Sweden

Corresponding Author’s e-mail: johan.liu@chalmers.se

\begin{abstract}
This paper presents a novel nanocomposite thermal interface material (Nano-TIM) consisting of a silver coated polyimide network and the indium matrix. One of the potential applications of this Nano-TIM is for heat dissipation in integrated circuits and electronic packaging. The shear strength of the Nano-TIM was investigated with DAGE-4000PSY shear tester. The shear strength of Nano-TIM is $4.5 \mathrm{MPa}$, which is $15 \%$ higher than that of the pure indium thermal interface material. The microstructure of cross-section and fracture surface was studied using Scanning Electron Microscopy (SEM). SEM pictures show a uniform polymer fiber distribution and solid interface between silver coated fibers and indium matrix. The thermal fatigue resistance of the Nano-TIM was evaluated by monitoring the variation of thermal interface resistance during the thermal cycling test $\left(-40\right.$ to $\left.125{ }^{\circ} \mathrm{C}\right)$. The thermal interface resistance was measured with a commercial xenon flash instrument after 100, 200, 300, 400, 500, and 1000 temperature cycles. The results of thermal cycling test show that Nano-TIM presented consistent reliability performance with pure indium. Furthermore, the cooling effect of Nano-TIM was demonstrated through measuring the power chip temperature in the die attached structure by using an Infrared Camera. In the test, the Nano-TIM shows a comparable cooling effect to pure indium TIM for die attach applications in electronics packaging.
\end{abstract}

\section{Introduction}

The development of small feature sizes and high performance electronic products has resulted in the tremendous increase of the chip power density. Thermal management, maintaining a low system temperature under a high power density loading, thus becomes a critical issue in the electronic packaging field. Some challenges in thermal management need to be addressed urgently. For example, the high thermal interface resistance between heat sink and silicon chip, generally caused by the surface roughness, could significantly reduce the efficiency of heat transfer. Therefore, thermal interfaces have been identified as one of the main bottlenecks in heat dissipation [1]. Currently, one effective solution for decreasing the thermal interface resistance is to fill the gap at the interface between silicon chip and heat sink by using a thermal interface material with high thermal and mechanical performance.

The current trends of developing new TIMs are focused on the nanocomposite materials, such as embedding carbon nanotubes [2]-[4] and silver nanowire arrays in a polymer matrix [5], due to the unique properties of nanomaterials on surface area, defects, mechanical, electrical, and 
thermal performance. Meanwhile, the improvement and characterization of traditional TIMs, including solder-based TIMs [6] and phase change materials [7], are also an important topic in thermal management field. The researchers have developed several metal based nano-fiber matrix composite TIMs for heat dissipation in the electronics packaging. L Zhang et al. reported the performance of indium-tin-bismuth alloy based nano-fiber composite TIMs used as a kind of phase change material [8]. However, phase change TIM material can easily be oxidation and corrosion at the elevated temperature cycles[9]. C Zandén et al. studied the thermal performance and reliability of tin-silver-copper based nano-polymers composite in a die attachment [10]. M Murugesan et al. developed the carbon fiber based tin-silver-copper alloy matrix composite TIM for thermal management of high power electronics [11].However, this kind of solder based nanocomposite TIM has to be soldered onto the substrate with around $220^{\circ} \mathrm{C}$ which possibly bring a risk of reliability of some temperature sensitive device[12].

In this paper, we choose indium as based material to develop TIM due to the indium's high thermal conductivity and low melting temperature[13]. However, pure indium is easily deformed due to a low yield strength and also have a risk of flowing out during die attach process[14]. Moreover, with its large coefficient of thermal expansion (compared to Si chip and heat sink) could be a potential challenge to strength and thermal fatigue resistance of an indium TIM. Therefore, addressing the weakness of indium, a novel nanocomposite thermal interface material (Nano-TIM) which consists of metallic indium and a silver coated polyimide (PI) network was developed. The polymer network defines the geometry of TIM, such as the shape and bond line thickness. Meanwhile, the metallic part is responsible for heat transfer. Importantly, nanofiber network can potentially improve the mechanical performance and reliability in microelectronics packaging[15][16]. The nano-sized polyimide fibers in the Nano-TIM can constrain the dislocation and delay the crack propagation, resulting in the enhanced strength and thermal fatigue resistance. The work in this paper focuses on characterizing the mechanical and thermal performance of the Nano-TIM for die attachment application in the electronics packaging. Moreover, the thermal cycling reliability of the Nano-TIM is also investigated. Finally, the real cooling effect of the Nano-TIM in high power chip packaging is tested using an Infrared (IR) camera system.

\section{Experimental procedure}

\subsection{Nano-TIM preparation}

The processes of the Nano-TIM preparation can be shown in three steps. Firstly, the polyimide nanofiber network is fabricated by using electrospinning technology. Electrospinning solution is consisted with 16wt\% PI dissolved in N, N-dimethylacetamide (DMAC). Electrospinning is performed using $18 \mathrm{kV}, 2 \mathrm{~mL} / \mathrm{h}$ feed rate and $20 \mathrm{~cm}$ between cannula and collector in a commercial electrospinning unite (Nanofiber Electrospinning Unite, Kato Tech). Secondly, surface modification of the nanofiber is performed to coat Ag layer onto the nanofiber for later metal infiltration process. The nanofiber network like a mesh is immersed in $4 \mathrm{M} \mathrm{KOH}$ aqueous solution at $80^{\circ} \mathrm{C}$ during 30 minutes to induce imide-ring cleavage and form potassium polyamate layer in the polymer chains on surface of nanofiber. Hereafter, the nanofibers are put in $100 \mathrm{mM}$ AgNO3 aqueous solution at $25^{\circ} \mathrm{C}$ for 30 minutes to facilitate an ion exchange process where the $\mathrm{Ag}^{+}$ions replaced potassium ions existing on the surface of nanofiber. Then $1 \mathrm{mM}$ DMAB aqueous solution is used to reduce $\mathrm{Ag}^{+}$ions to form a silver layer on the fiber surface. Finally, liquid indium is infiltrated in this Ag coated PI network by using an infiltration machine. The detailed description of fabrication processes, such as electrospinning and infiltration, are found in 
previous papers by the authors [17]-[20]. Ag-coated PI network and a piece of Nano-TIM are shown in Figure 1. After the infiltration process, the fibers are covered by indium, being invisible from the top surface. The size of Nano-TIM can be controlled during infiltration process. In this study, $50 \mu \mathrm{m}$ thick Nano-TIM with $3 \mathrm{~cm}$ diameter is selected for performance tests.

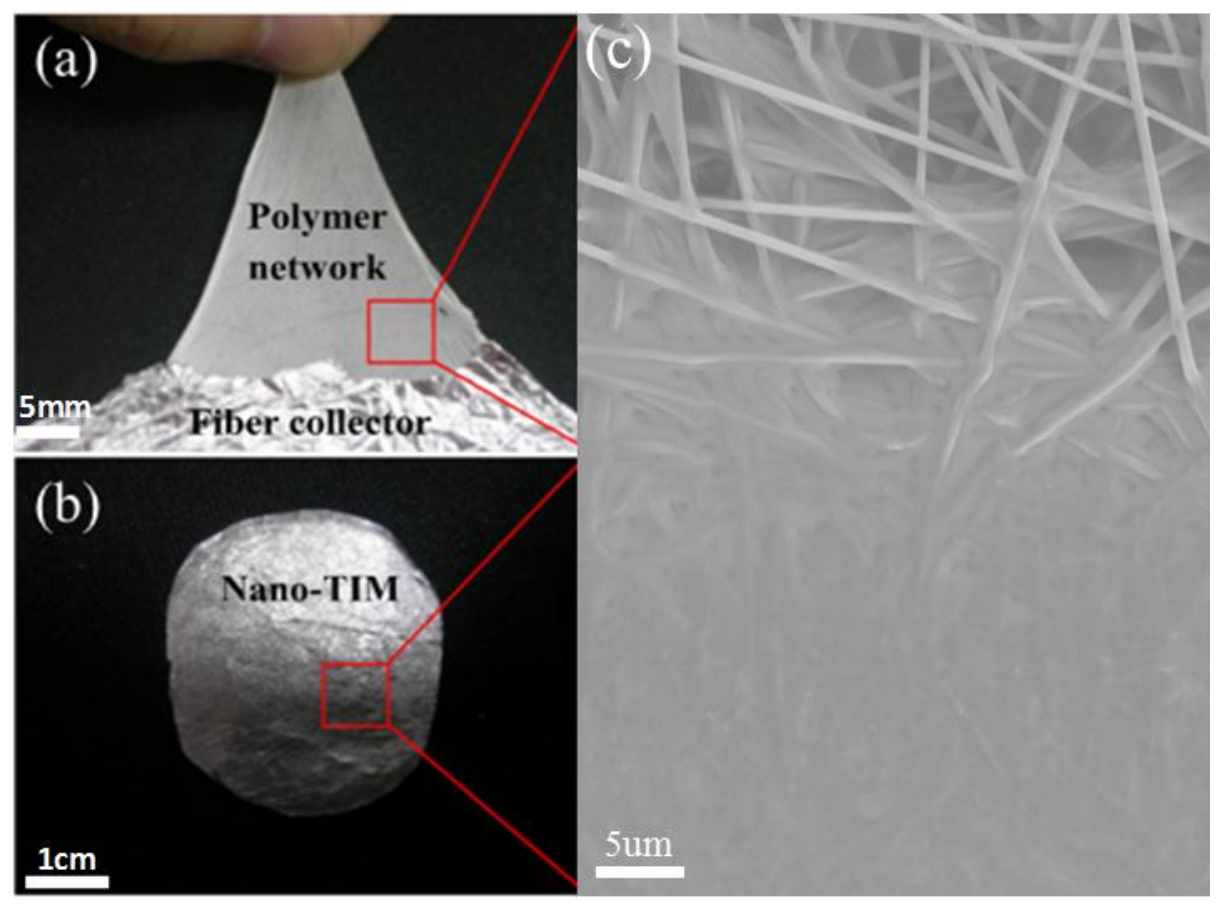

Figure 1. (a) Polyimide network formed with electrospinning process; (b) a piece of Nano-TIM fabricated with infiltration process; (c) SEM image of nanofiber network after partly metal infiltration.

\subsection{Shear test}

The shear test samples are designed into the sandwich structure as shown in Figure 2(a). A piece of Nano-TIM is firstly placed on a $\mathrm{Cu}$ pad with an electroless nickel immersion gold (ENIG) surface finish. A $2 \times 2 \mathrm{~mm}$ silicon chip with titanium /gold (20/100 nm) surface finish was then pressed on the Nano-TIM at $180{ }^{\circ} \mathrm{C}$ with $0.4 \mathrm{MPa}$ of pressure for 1.5 minutes. After that, this sandwich structure is cooled down to the room temperature in the air. The shear test on this sandwich structure is executed using a DAGE-4000PSY shear tester. The shear speed and height are set to $200 \mu \mathrm{m} / \mathrm{s}$ and $100 \mu \mathrm{m}$ respectively. In order to evaluate the effect of the fibers on the Nano-TIM, pure indium TIM which went through the same process with the Nano-TIM, is also tested with the same sample geometry, and 10 samples were prepared and tested for each case.

(a)

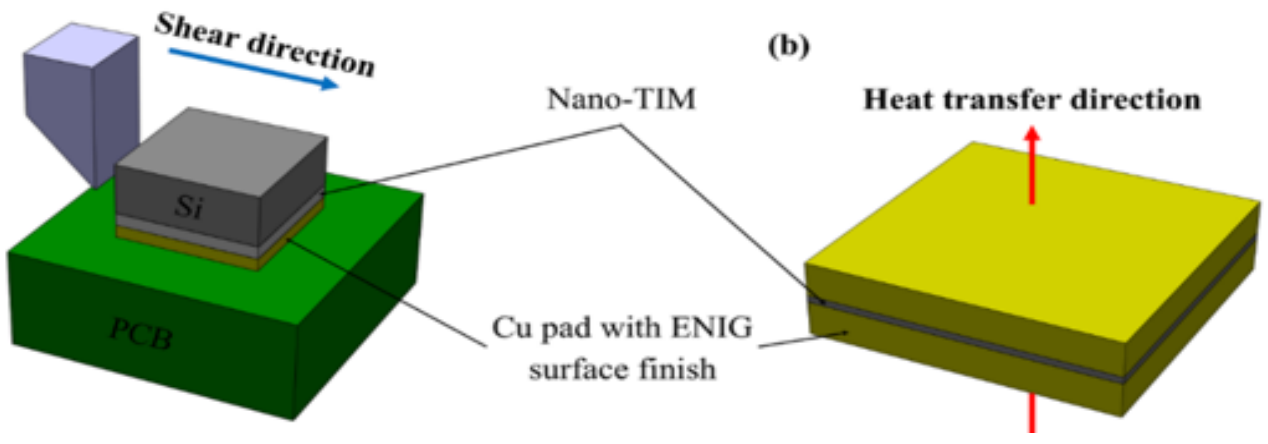


Figure 2. The schematic structures of the samples for (a) shear test and (b) thermal interface resistance measurement.

\subsection{Thermal interface resistance measurement}

The sample structure for thermal interface resistance measurement is shown in Figure 2(b). A piece of Nano-TIM is sandwiched between two $8 \times 8 \mathrm{~mm}^{2}$ copper/ENIG pieces and reflowed with the same processes as shear test sample. The pure indium samples are also prepared as reference. In total 5 samples are prepared for each case. The thermal interface resistance is measured by using a commercial xenon flash instrument (LFA447, Netzsch).

\subsection{Thermal cycling test}

After thermal interface resistance measurement, all samples are transferred to an environmental test chamber (924E, Despatch) for the thermal cycling (TC) test. The temperature range of the TC test was set from -40 to $115^{\circ} \mathrm{C}$, with the duration time per cycle and the soak time being 30 and 10 minutes respectively. The TC test is paused at 100, 200, 300, 400, 500, and 1000 cycles in order to check the variation of the thermal interface resistance.

\subsection{Thermal Infrared Camera Test}

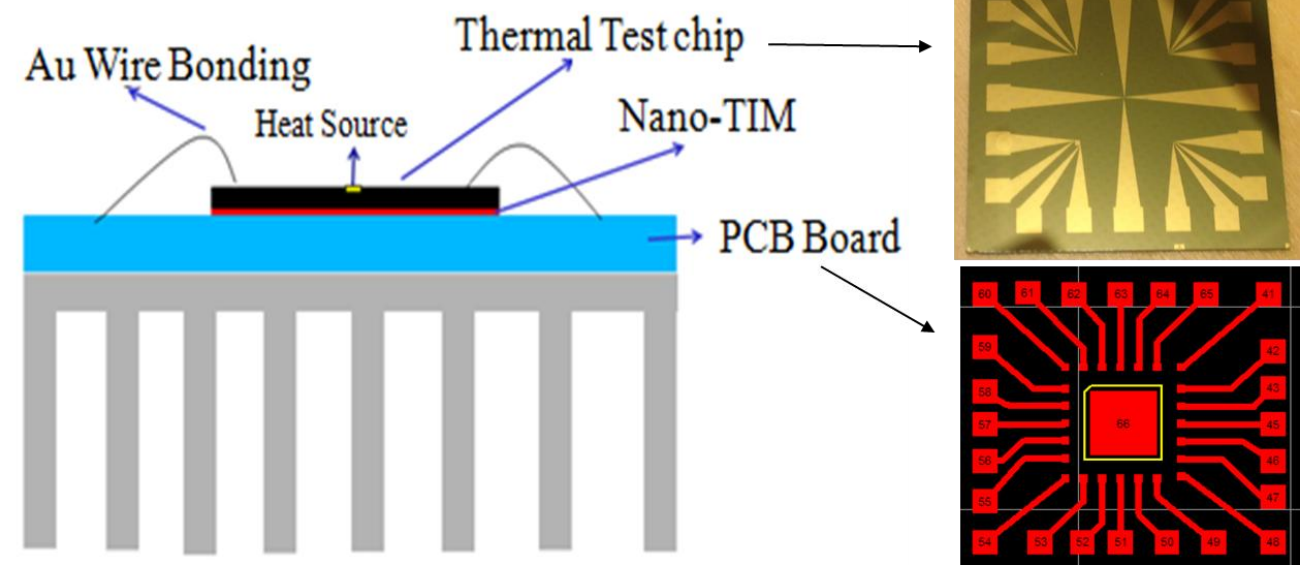

Figure 3. Infrared Camera test setup for detecting heat distribution

The heat dissipation effect of the Nano-TIM for die attach applications is investigated by using a thermal infrared camera. The test setup is shown in Figure 3. The power chip with hot spot is attached onto a copper heat sink with TIM materials by using the same process as the shear test samples. The infrared camera (FLIR SC660) is then used to test the heat spreading map of the power chip with 10 Watt loading. The hot spot temperature and heat distribution of the power chip are captured by infrared camera when the samples reached thermal equilibrium after 2 min. 


\section{Results and Discussion}

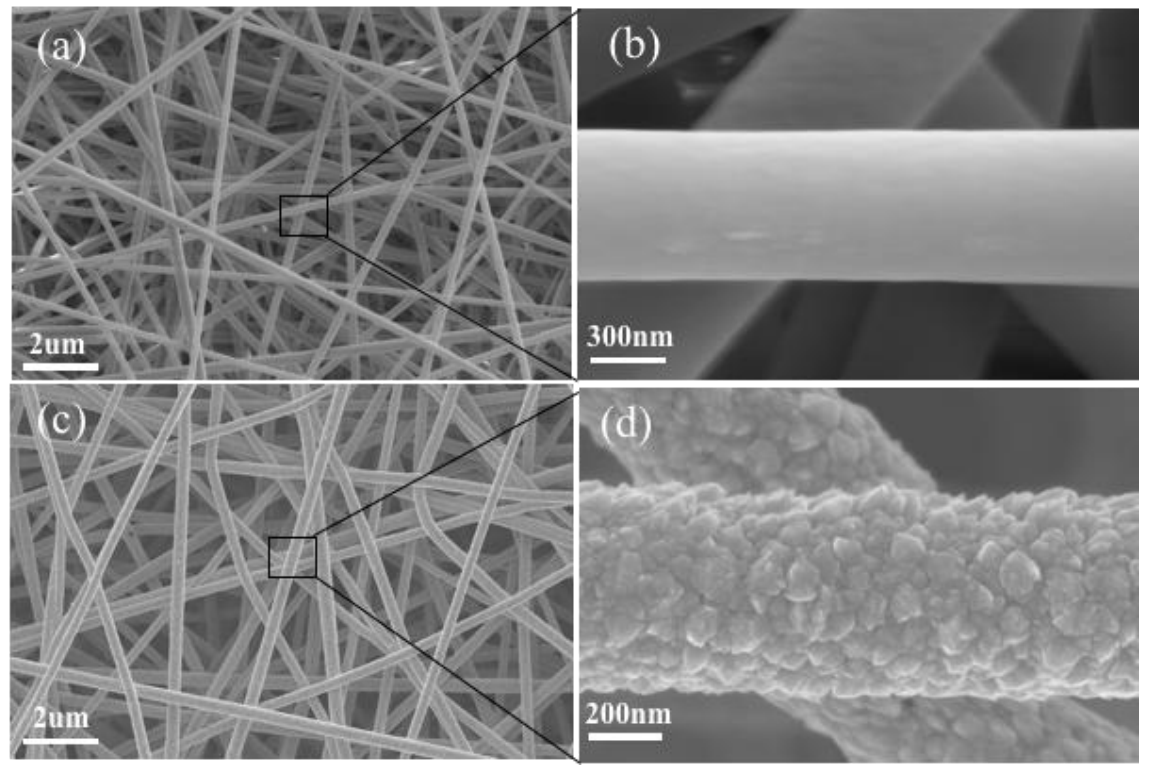

Figure 4. (a) and (b) show pristine polyimide nanofibers network formed through electrospinning process; (c) and (d) show the nanofibers network with Ag nanoparticles coating after surface modification.

The structure details of polyimide nanofibers network before and after surface modification are summarized in the Figure 4. From the image analysis, the mean fiber diameter is found to be approximately $500 \pm 200 \mathrm{~nm}$. After surface modification process, a layer of silver coated on the nanofiber surface is observed in the Figure 4(c) and (d). And the coverage of silver layer is uniformly and completely coated on the whole nanofibers. The function of this silver layer is to act as interfacial layer between the low surface energy polymer fibers and indium metal to facilitate metal infiltration. Moreover, through comparing the overall structure of 3D fiber network between Figure 4(a) and Figure 4(c), the surface modification does not bring any apparent damaging effect on the overall structure.

In order to study the inner structure of Nano-TIM, the cross-section samples are prepared. Here the pure indium is used as a reference. Pure indium at the interface between the TIM and $\mathrm{Cu} / \mathrm{ENIG}$ pad is shown in Figure 5(a). Some $\mathrm{Si}$ pieces are found in the indium matrix. These $\mathrm{Si}$ pieces come from the Si chip used during the cross-section making process. The Si pieces were polished off the Si chip and embedded in the soft indium. In Figure 5(b), the polymer fibers can be observed in the indium matrix. There are no visible voids that can be detected in Figure 5(b), indicating the porous polymer network has been completely filled with indium after the infiltration process. Figure 5(c) shows an image of Nano-TIM with high magnification. In Figure 5(c), each fiber is tightly covered by the indium matrix. In general, the porous PI network is extremely difficult to be fully wetted and filled by liquid indium due to the inert nature of PI and the small gap between the PI fibers as shown in the Figure 5(e). However, in this Nano-TIM, the surface of the PI fibers has been coated by silver, which significantly improves the wettability of liquid indium to the PI surface. For all nanofibers observed, each individual nanofiber was tightly surrounded by indium under the help of the silver particle as shown in the representative image 
given in Figure 5(d). As a result, the liquid indium can successfully infiltrates the PI network by using high pressure in the infiltration process.
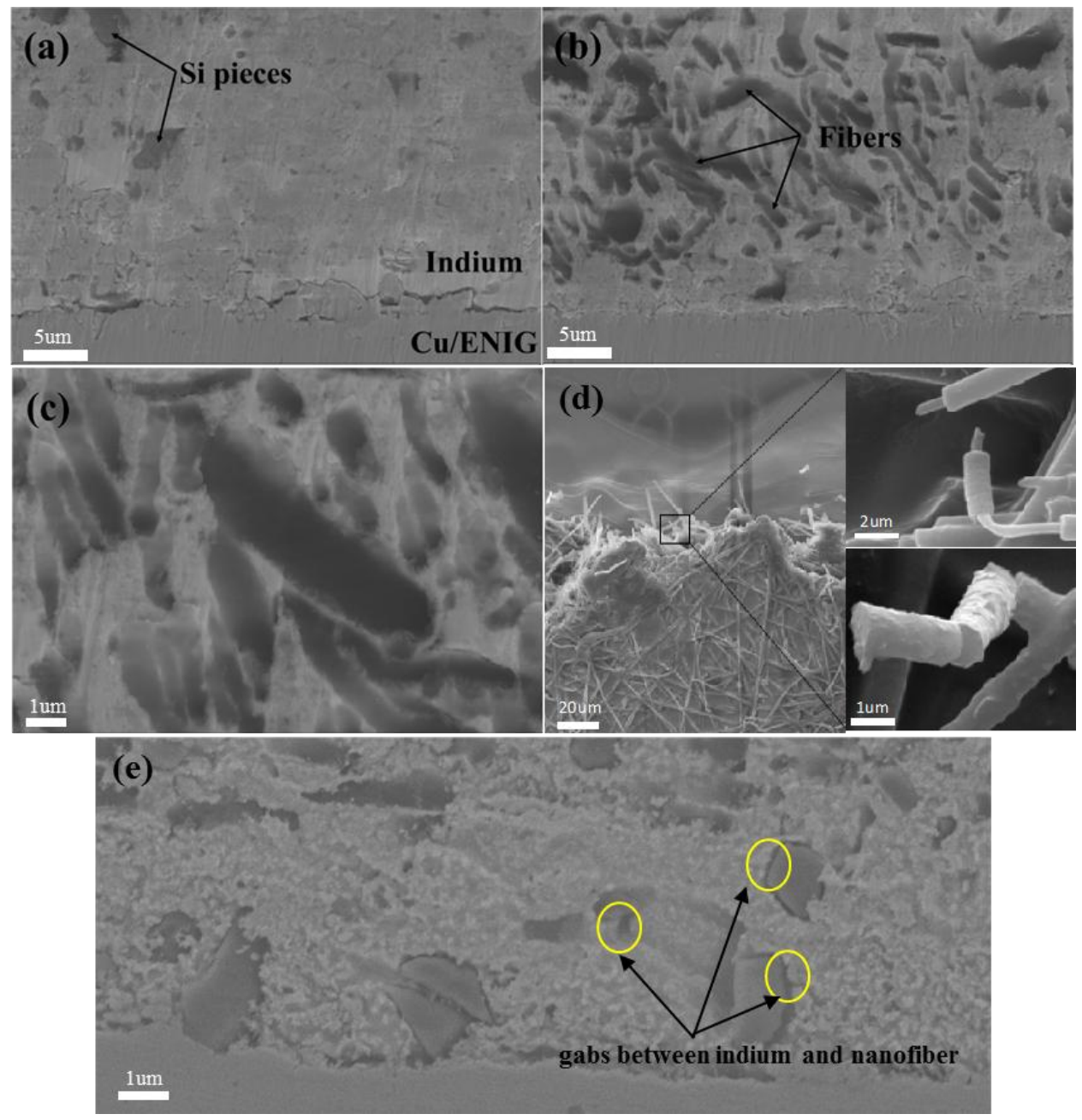

Figure 5. Cross-sections of (a) pure indium and (b) Nano-TIM with Cu/ENIG pad; (c) is a high magnification image of the Nano-TIM; (d) shows the inner structure of Nano-TIM after damage; (e) shows the interface status between indium and the nanofiber without silver coating. 


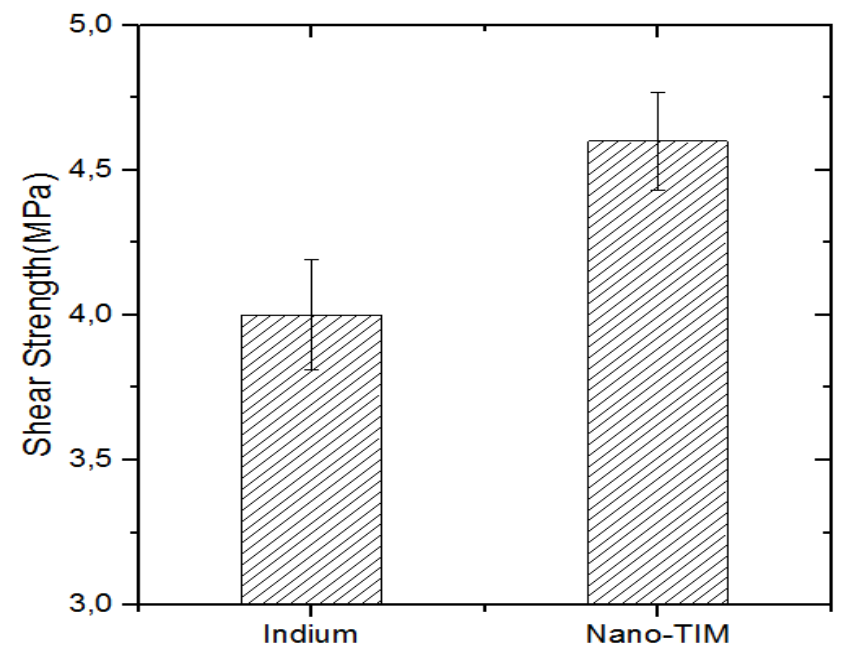

Figure 6. The mechanical strength performance of Nano-TIM and pure indium.

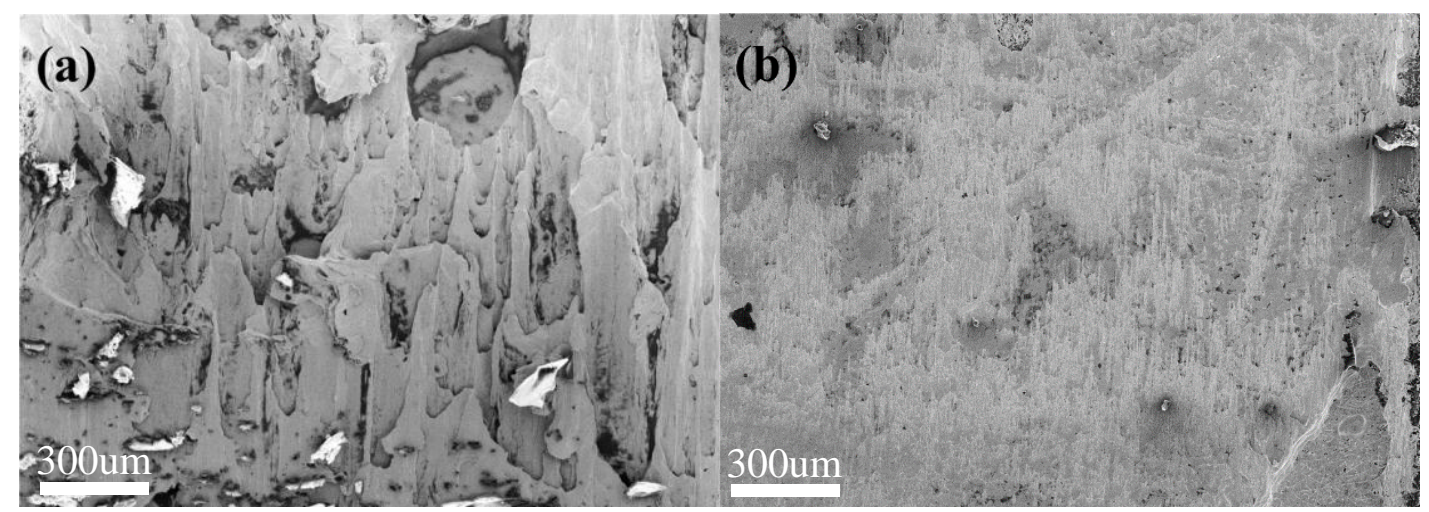

Figure 7. Fracture surface of (a) pure indium (a) and Nano-TIM (b) after shear test.

Figure 6 shows the results of shear test for Nano-TIM and pure indium samples. The average shear strength of Nano-TIM is higher than that of pure indium, which could be attributed to the reinforcement effect of silver coated PI fibers. The dislocation motion in the Nano-TIM can be impeded by those Ag coated fibers, which limits the crack initiation and propagation during shear test and subsequently increases the shear strength of pure indium. To verify this point, the fracture surface after the shear test was also studied. The fracture surface of pure indium in Figure 7(a) is composed of dimples, showing a typical ductile fracture mode. In Figure 7(b), the fracture surface of Nano-TIM also shows the ductile fracture mode, but the microstructure is much finer than that of pure indium. Comparing to the pure indium, the dimple size and elongation of the Nano-TIM is also smaller. This fine microstructure of the fracture surface of Nano-TIM could be caused by the PI network which separates the large indium matrix to small areas. This finer microstructure of fracture surface of Nano-TIM directly justified that the nanofibers do limit the crack initiation and propagation during the shear test. 


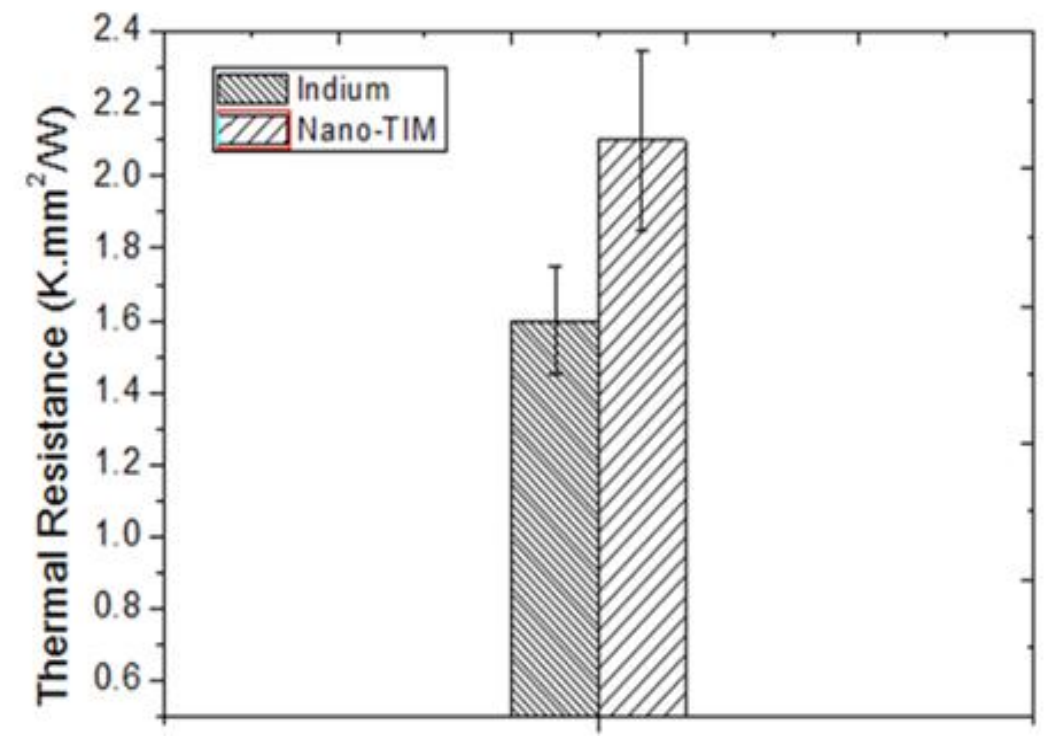

Figure 8 . The thermal interface resistance of Nano-TIM and pure indium.
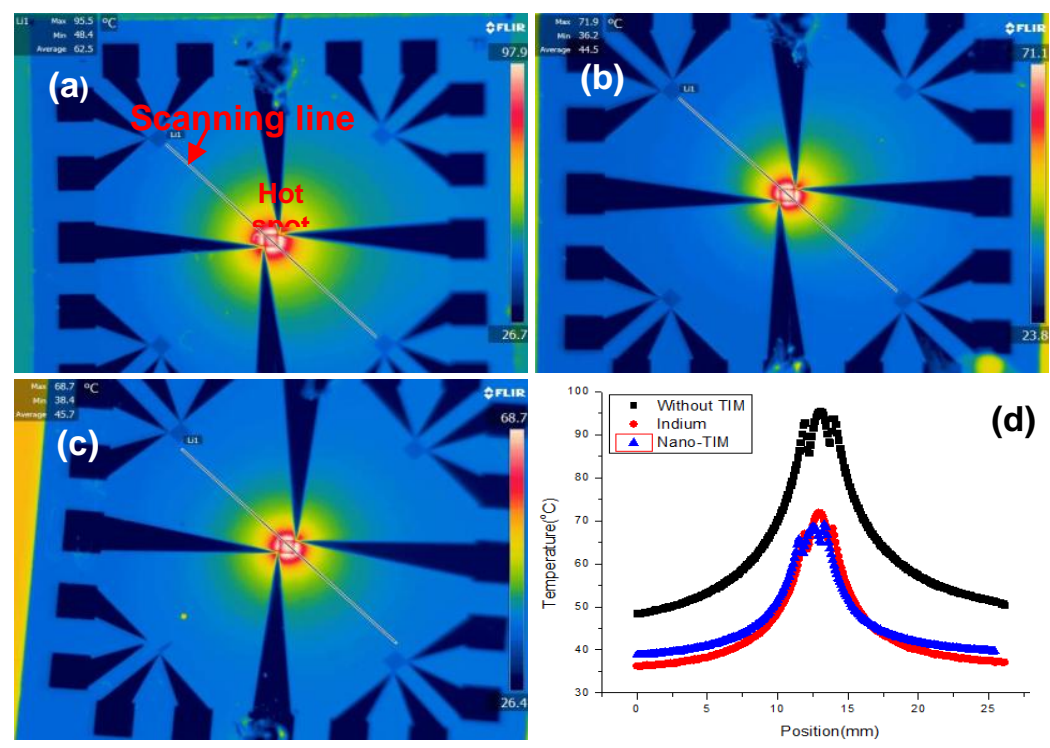

Figure 9. IR test results: temperature distribution of the chip (a) without TIM, (b) with pure indium, and (c) with the Nano-TIM; (d) Temperature distribution along the line direction shown in Figure 9 (a, b, c).

For investigating the heat dissipation performance of Nano-TIM in application, thermal interface resistance of pure indium and Nano-TIM is measured respectively. And the results are showed in Figure 8. The average thermal interface resistance of Nano-TIM in the vertical direction is 2.1 $\mathrm{K} \cdot \mathrm{mm}^{2} \cdot \mathrm{W}^{-1}$, which is higher than that of pure indium $\left(1.7 \mathrm{~K} \cdot \mathrm{mm}^{2} \cdot \mathrm{W}^{-1}\right)$. In order to investigate heat dissipation effect of this thermal interface resistance difference in real application, the thermal infrared camera is used to capture the temperature distribution of power chip as shown in 
Figure 9. Comparing Figure 9(b) with (c), the temperature distribution of Nano-TIM sample is almost the same as that of pure indium TIM sample. The similar temperature distribution between pure indium and Nano-TIM indicates that the PI network in the indium matrix would not noticeably degrade on the thermal performance of indium matrix and bonding quality at the interface. The Nano-TIM developed in this study can be roughly categorized to a kind of metal matrix composite material. In the metal matrix composite material, the thermal conductivity mainly depends on the movement of electrons in the metallic matrix. In the Nano-TIM, the silver coating of PI fibers promotes the formation of a solid interface between the fiber and the matrix, retaining the continuous structure of the indium matrix. That could be the reason why the NanoTIM keep the same heat dissipation effect as pure indium in the application. On the other hand, the nanofibers network may act like a mesh to define the geometry and prevent the melting metal flow away and splash during reflow process. This is also a possible reason why the Nano-TIM sample have almost the same cooling effect as pure indium sample although the Nano-TIM shows a little bit higher thermal resistance than indium in the previous measurement results. As a reference, the hot spot area of the chip without TIM is also tested, which shows obviously larger than the chip with TIM. Figure 9(d) shows that the maximum temperature of the chip without TIM is almost $30{ }^{\circ} \mathrm{C}$ higher than two other chips with TIM, showing how much effect the TIM has in increasing heat dissipation efficiency.

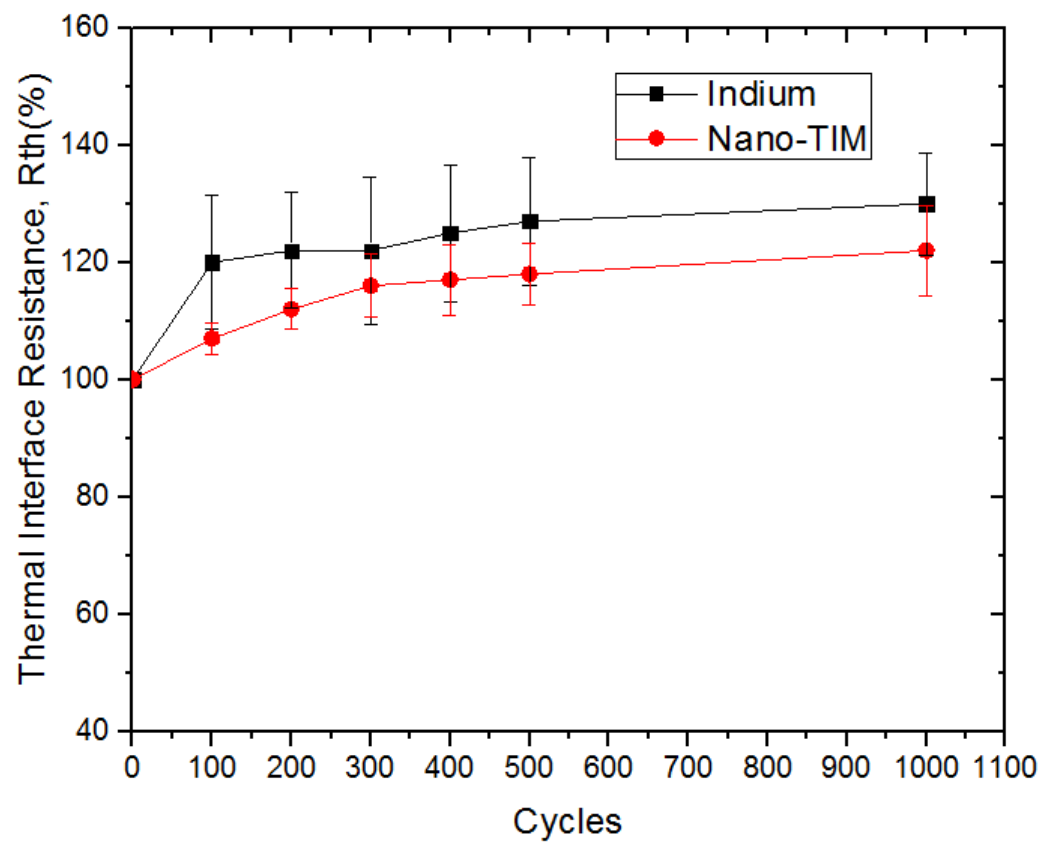

Figure 10. Thermal interface resistance measurement after 100, 200, 300, 400, 500, and 1000 temperature cycles.

In order to evaluate the change in thermal performance and long-term reliability of nano-TIM, we carried out thermal cycling under harsh conditions $\left(-40\right.$ to $115^{\circ} \mathrm{C} @ 2$ cycles per hour $)$ while periodically monitoring the change in thermal resistance. The results are presented in Figure 10. The overall trend shows that the thermal interface resistance of two types of samples were both 
slightly increased with the thermal cycles increasing. After 1000 cycles, the Nano-TIM and indium samples both do not have drastically change in the thermal performance, which indicate that the Nano-TIM have comparably stable reliability with indium. The slight increase of thermal interface resistance may be attributed to the formation of small cracks in the structure which is caused by harsh cycling condition. The coefficient of thermal expansion mismatch between different materials will lead to the stress concentration at initial cracks and promote the propagation of these cracks with temperature cycles[21]. Therefore, the change percentage of thermal interface resistance of the samples both reach to the maximum around $20 \%$ at the 1000 cycles. Considering the standard error bar overlap, the change range of thermal interface resistance of Nano-TIM is consistent with indium sample, which indicates that Nano-TIM still can keep as high bonding quality as pure indium after 1000 cycles.

\section{Conclusions}

Shear strength, thermal interface resistance, thermal fatigue resistance and cooling effect of a dieattach structure using a Nano-TIM were investigated in this paper. Increased shear strength of Nano-TIM is attributable to the reinforcement effect of silver coated PI fibers on the indium matrix. The silver coated PI fibers distributed in the indium matrix impede the crack propagation and consequently improve the mechanical performance of pure indium TIM. Mechanical failure doesn't occur in both the indium and Nano-TIM materials after 1000 temperature cycles. NanoTIM shows comparably stable reliability with indium. Moreover, the heat dissipation ability of Nano-TIM in the die attach structure is comparable to that of pure indium, indicating that the Agcoated PI polymer network is able to improve the mechanical performance of pure indium whilst avoiding the degradation of thermal performance and interface bonding quality.

\section{Acknowledgments}

This work was supported by the EU program"Smartpowerand the Sustainable Production Initiative and the Production Area of Advance at Chalmers. The authors are grateful for the support of the Swedish National Science Foundation (VR) under the projects "Thermoelectric Nanostructures for On-Chip Cooling" (EM11-0002) and "Carbon Based 3D GaN high Speed Electronics Systems", (SE13-0061) as well as the financial support of the NSFC program (51272153) and STC program (12JC1403900).

\section{References}

[1] ITRS, "International technology roadmap for semiconductors 2013 edition - assembly and packaging," tech. rep., 2013.

[2] H. Huang, C. H. Liu, Y. Wu, and S. Fan, "Aligned Carbon Nanotube Composite Films for Thermal Management," Adv. Mater., vol. 17, no. 13, pp. 1652-1656, 2005.

[3] B. A. Cola, S. L. Hodson, X. Xu, and T. S. Fisher, "Carbon Nanotube Array Thermal Interfaces Enhanced With Paraffin Wax," pp. 765-770, Jan. 2008.

[4] E. S. Choi, J. S. Brooks, D. L. Eaton, M. S. Al-Haik, M. Y. Hussaini, H. Garmestani, D. Li, and K. Dahmen, "Enhancement of thermal and electrical properties of carbon nanotube polymer composites by magnetic field processing," J. Appl. Phys., vol. 94, no. 9, pp. 6034-6039, Nov. 2003.

[5] J. Xu, A. Munari, E. Dalton, A. Mathewson, and K. M. Razeeb, "Silver nanowire array-polymer composite as thermal interface material," J. Appl. Phys., vol. 106, no. 12, p. 124310, Dec. 2009.

[6] C. Deppisch, T. Fitzgerald, A. Raman, F. Hua, C. Zhang, P. Liu, and M. Miller, "The material optimization and reliability characterization of an indium-solder thermal interface material for CPU packaging," JOM, vol. 58, no. 6, pp. 67-74, Jun. 2006. 
[7] C. Ramaswamy, S. Shinde, F. Pompeo, W. Sablinski, and S. Bradley, "Phase change materials as a viable thermal interface material for high-power electronic applications," in The Ninth Intersociety Conference on Thermal and Thermomechanical Phenomena in Electronic Systems, 2004. ITHERM '04, 2004, vol. 2, pp. 687-691 Vol.2.

[8] L. Zhang, X. Luo, X. Lu, and J. Liu, "Study on the Reliability of Nano-Structured Polymer-Metal Composite for Thermal Interface Material," ECS Trans., vol. 34, no. 1, pp. 991-995, Mar. 2011.

[9] F. Sarvar, D. C. Whalley, and P. P. Conway, "Thermal Interface Materials - A Review of the State of the Art," in Electronics Systemintegration Technology Conference, 2006. 1st, 2006, vol. 2, pp. 12921302.

[10] J. Liu, C. Zandén, X. Luo, and L.-L. Ye, "A new solder matrix nano polymer composite for thermal management and die attach applications," Compos. Sci. Technol., vol. 94, pp. 54-61, 2014.

[11] M. Murugesan, C. Zandén, X. Luo, L. Ye, V. Jokubavicius, M. Syväjärvi, and J. Liu, "A carbon fiber solder matrix composite for thermal management of microelectronic devices," J. Mater. Chem. C, vol. 2, no. 35, pp. 7184-7187, Aug. 2014.

[12] "Reliability challenges in 3D IC packaging technology." [Online]. Available: http://www.sciencedirect.com/science/article/pii/S0026271410005214. [Accessed: 24-Sep-2015].

[13] X. C. T. Ph.D, Advanced Materials for Thermal Management of Electronic Packaging. Springer Science \& Business Media, 2011.

[14] J. L. F. Goldstein and J. W. Morris, "Microstructural development of eutectic Bi-Sn and eutectic In-Sn during high temperature deformation," J. Electron. Mater., vol. 23, no. 5, pp. 477-486, May 1994.

[15] M. P. Renavikar, N. Patel, A. Dani, V. Wakharkar, G. Arrigotti, V. Vasudevan, O. Bchir, A. P. Alur, C. K. Gurumurthy, and R. W. Stage, "Materials technology for environmentally green micro-electronic packaging," Intel Technol J, vol. 12, no. 1, pp. 1-15, 2008.

[16] F. Hua and C. Deppisch, "Solder as thermal interface material for high power devices," SMTA NEWS J. Surf. Mt. Technol., vol. 19, no. 1, p. 21, 2006.

[17] B. Carlberg, T. Wang, J. Liu, and D. Shangguan, "Polymer-metal nano-composite films for thermal management," Microelectron. Int., vol. 26, no. 2, pp. 28-36, 2009.

[18] B. Carlberg, J. Liu, and L.-L. Ye, "Polymer nanofiber based continuous metal phase composite for thermal management applications," in Electronic System-Integration Technology Conference (ESTC), 2010 3rd, 2010, pp. 1-6.

[19] B. Carlberg, L.-L. Ye, and J. Liu, "Surface-Confined Synthesis of Silver Nanoparticle Composite Coating on Electrospun Polyimide Nanofibers," Small, vol. 7, no. 21, pp. 3057-3066, Nov. 2011.

[20] B. Carlberg, L.-L. Ye, and J. Liu, "Polymer-metal nanofibrous composite for thermal management of microsystems," Mater. Lett., vol. 75, pp. 229-232, May 2012.

[21] E. J. Cheng and Y.-L. Shen, "Thermal expansion behavior of through-silicon-via structures in threedimensional microelectronic packaging," Microelectron. Reliab., vol. 52, no. 3, pp. 534-540, Mar. 2012. 